\title{
Mode of action of nintedanib in the treatment of idiopathic pulmonary fibrosis
}

\author{
Lutz Wollin ${ }^{1}$, Eva Wex ${ }^{1}$, Alexander Pautsch¹, Gisela Schnapp ${ }^{1}$, \\ Katrin E. Hostettler ${ }^{2}$, Susanne Stowasser ${ }^{3}$ and Martin Kolb ${ }^{4}$
}

\author{
Affiliations: \\ ${ }_{1}^{1}$ Boehringer Ingelheim Pharma GmbH \& Co. KG, Biberach, Germany. \\ ${ }^{2}$ University Hospital Basel, Basel, Switzerland. \\ ${ }^{3}$ Boehringer Ingelheim Pharma GmbH \& Co. KG, Ingelheim am Rhein, Germany. \\ ${ }^{4}$ McMaster University, Hamilton, ON, Canada.
}

\section{Correspondence:}

Stefan-Lutz Wollin, Boehringer Ingelheim Pharma GmbH \& Co KG, Birkendorfer Strasse 65, 88397 Biberach, Germany. E-mail: Stefan-lutz.wollinaboehringer-ingelheim.com

ABSTRACT Idiopathic pulmonary fibrosis (IPF) is a progressive and ultimately fatal disease characterised by fibrosis of the lung parenchyma and loss of lung function. Although the pathogenic pathways involved in IPF have not been fully elucidated, IPF is believed to be caused by repetitive alveolar epithelial cell injury and dysregulated repair, in which there is uncontrolled proliferation of lung fibroblasts and differentiation of fibroblasts into myofibroblasts, which excessively deposit extracellular matrix (ECM) proteins in the interstitial space. A number of profibrotic mediators including platelet-derived growth factor (PDGF), fibroblast growth factor (FGF) and transforming growth factor- $\beta$ are believed to play important roles in the pathogenesis of IPF. Nintedanib is a potent small molecule inhibitor of the receptor tyrosine kinases PDGF receptor, FGF receptor and vascular endothelial growth factor receptor. Data from in vitro studies have shown that nintedanib interferes with processes active in fibrosis such as fibroblast proliferation, migration and differentiation, and the secretion of ECM. In addition, nintedanib has shown consistent anti-fibrotic and anti-inflammatory activity in animal models of lung fibrosis. These data provide a strong rationale for the clinical efficacy of nintedanib in patients with IPF, which has recently been demonstrated in phase III clinical trials.

@ERSpublications

Nintedanib interferes with processes active in fibrosis, e.g. fibroblast proliferation, migration and differentiation http://ow.ly/Iae9z

\footnotetext{
This article has supplementary material available from erj.ersjournals.com

Received: Sept 232014 | Accepted after revision: Jan 05 2015 | First published online: March 052015

Support statement: This article and the studies of nintedanib described in this article were funded by Boehringer Ingelheim. Funding information for this article has been deposited with FundRef.
}

Conflict of interest: Disclosures can be found alongside the online version of this article at erj.ersjournals.com

Copyright OERS 2015 ERJ Open articles are open access and distributed under the terms of the Creative Commons Attribution Non-Commercial Licence 4.0. 


\section{Introduction}

Nintedanib (international non-proprietary name), formerly known by its development code BIBF 1120 , is a small molecule that was originally designed as an ATP-competitive inhibitor of fibroblast growth factor receptor (FGFR)-1 and vascular endothelial growth factor receptor (VEGFR)-2. Both these receptors are pro-angiogenic receptor tyrosine kinases and nintedanib was designed as an anti-angiogenic drug for cancer indications. The clinical development of nintedanib for cancer indications, including nonsmall cell lung cancer, colorectal cancer and ovarian cancer, is ongoing. As nintedanib is also an inhibitor of platelet-derived growth factor receptor (PDGFR)- $\alpha$ and $\beta$, it was selected for development as a potential treatment for idiopathic pulmonary fibrosis (IPF). The fact that the pathobiology of IPF shows several striking similarities and links to cancer biology (reviewed in [1]) also supports the rationale for the exploration of nintedanib in IPF. In the two replicate phase III INPULSIS ${ }^{\oplus}$ studies [2], nintedanib was shown to slow disease progression in patients with IPF by reducing the annual rate of decline in forced vital capacity (FVC) [3]. This review summarises the current understanding of the mode of action of nintedanib in fibrotic lung diseases like IPF. The roles of the main target receptor tyrosine kinases of nintedanib in IPF are explained. In vitro experiments with primary lung fibroblasts from patients with IPF and from control donors, and in vivo studies in animal models of lung fibrosis, are summarised. Taken together, these studies demonstrate the potent anti-fibrotic and anti-inflammatory activities of nintedanib. These features may explain the slowing of disease progression demonstrated in patients with IPF treated with nintedanib.

\section{Idiopathic pulmonary fibrosis}

IPF is a devastating and disabling progressive lung disease associated with a median survival of only 2-3 years after diagnosis [4]. IPF is the most common form of the idiopathic interstitial pneumonias [5], with an incidence ranging between 0.22 and 17.4 per 100000 population depending on the case definition, the region and the methodology used [6]. IPF usually presents in the sixth or seventh decade of life and occurs more frequently in men than in women $[4,7]$. In international guidelines for the management of IPF, published in 2011 [4], the treatment options that were strongly recommended were limited to oxygen supplementation and lung transplantation. Despite high medical need, these guidelines did not recommend any specific pharmacological therapy for the chronic treatment of IPF [4]. Pirfenidone was introduced to the market for the treatment of IPF in Japan in 2008 and, based on the results of two phase III clinical trials (CAPACITY-1 and 2) [8], was later launched in several countries and regions including Europe, Canada, South Korea, China, India, Mexico and Argentina. An additional phase III study (ASCEND) was requested to support its regulatory registration in the USA and the positive results of this trial have recently been published [9]. With the publication of the phase III clinical results on the efficacy and safety of nintedanib in IPF (INPULSIS ${ }^{-}-1$ and INPULSIS ${ }^{\oplus}$-2) [3] and the recent approval of both pirfenidone and nintedanib for the treatment of IPF by the US Food and Drug Administration, physicians will soon have a choice of treatments for IPF after more than a decade of disappointment and failed clinical trials.

The pathology of IPF is characterised by repetitive microscopic alveolar epithelial cell injury and dysregulated repair, fibrosis and excessive deposition of extracellular matrix (ECM), resulting in loss of parenchymal architecture and lung function [10, 11]. The disease-inducing insult is unknown, but smoking, environmental and occupational exposures, viral infections, gastric acid reflux and genetic predisposition have been reported as risk factors for IPF [4, 7, 12, 13]. In IPF, fibroblasts exhibit unregulated proliferation and differentiate into myofibroblasts, which excessively produce ECM components. Myofibroblasts accumulated in clusters (fibroblastic foci) are considered the hallmark cells in the development of lung fibrosis [14]. The origin of myofibroblasts in pulmonary fibrosis is still controversial [15]. The pool in the fibrotic lung may be replenished through various mechanisms, including epithelial-mesenchymal transition (EMT) [16], fibrocyte recruitment [17], pericyte transdifferentiation [18], pleural mesothelial cells [19] or expansion of the local fibroblast population [20]. Apoptosis of the fibrotic cell pool in the lungs of patients with IPF is impaired and with it, the re-epithelialisation and restoration of the normal lung architecture [21].

The microcirculation in the lungs of patients with IPF is substantially altered. Not surprisingly for a disease characterised by patchy fibrosis close to normal lung parenchyma, these microvascular abnormalities are heterogeneous. Published reports from both human and experimental lung fibrosis indicate that fibrotic areas have fewer blood vessels, whereas adjacent non-fibrotic tissue is highly vascularised [22-25]. It remains to be clarified whether the increased capillary density in the least fibrotic regions is a compensatory response or whether neovascularisation plays a key role in the dysregulated matrix remodelling in IPF [26].

A multitude of profibrotic mediators, including interleukin (IL)-1 $\beta$, tumour necrosis factor- $\alpha$, connective tissue growth factor (CTGF), transforming growth factor (TGF)- $\beta$, IL-13, platelet-derived growth factor (PDGF) and fibroblast growth factor (FGF), and their signalling cascades are believed to play an important 
role in the pathogenesis of fibrotic lung diseases [27-29]. For example, IL-13 is a potent stimulator of fibroblast proliferation and ECM synthesis; it induces profibrotic cytokines, including TGF- $\beta$, PDGF and CTGF, and ECM components including collagen 1 and fibronectin [30]. TGF- $\beta 1$ has multiple functions: it promotes chemotaxis and proliferation of fibroblasts, differentiation of fibroblasts into myofibroblasts and EMT, and it protects myofibroblasts from apoptosis [30]. TGF- $\beta$ promotes the production of a range of profibrotic cytokines and tissue inhibitors of metalloproteinases (TIMPs) and inhibits matrix-degrading proteases [30]. The following sections will focus on the growth factors and growth factor receptors that are implicated in proliferation, migration and transdifferentiation of the fibroblast and myofibroblast pool in fibrotic lesions in IPF, and that represent targets for nintedanib.

\section{PDGF/PDGFR signalling in pulmonary fibrosis}

PDGF comprises a family of dimeric proteins made up of four polypeptide chains: A, B, C and D. These dimeric ligands interact with homodimers or heterodimers of PDGFR- $\alpha$ and $\beta$. The extracellular region of the receptor consists of five immunoglobulin-like domains while the intracellular part is a tyrosine kinase domain. Ligand binding leads to autophosphorylation of the receptors and subsequent downstream signalling via Ras, Raf, mitogen-activated protein kinase kinase (MEK), extracellular signal-regulated kinase (ERK) and phosphatidylinositol-4,5-bisphosphate 3-kinase (PI3K) (fig. 1) [28]. PDGF is a potent mitogen for fibroblasts [31] and appears to play an essential role in the expansion of myofibroblasts by stimulating proliferation, migration and survival [28]. When too active or too numerous, myofibroblasts deposit excessive connective tissue products in the interstitial space. The result is a distorted alveolar architecture with compromised gas exchange [4, 32]. In the human lung, PDGF is produced mainly by alveolar macrophages and epithelial cells $[28,33]$. Evaluation of alveolar macrophages from the lungs of patients with IPF demonstrated that these cells spontaneously release four times more PDGF than alveolar macrophages from control donors [34].

Animal models of lung injury with subsequent fibrosis have been invaluable in defining a role for PDGF in the progression of fibrosis. PDGFR-specific tyrosine kinase inhibitors reduce radiation-induced lung fibrosis in mice [35, 36], and vanadium pentoxide-induced pulmonary fibrosis in rats [37]. Imatinib, a PDGFR and c-Abl inhibitor, was shown to reduce bleomycin-induced lung fibrosis in mice [38, 39], and asbestos-induced interstitial pneumonia in mice [40]. However, in a phase II clinical trial in patients with IPF and mild-to-moderate impairment of lung function treated with imatinib or placebo for 96 weeks, imatinib did not affect survival or lung function [41]. Only a transient improvement in oxygenation was shown at week 48 [41]. These clinical results suggest that although PDGFR inhibition consistently reduces pulmonary fibrosis in animal models, PDGFR inhibition alone may not suffice to treat IPF.

\section{FGF/FGFR signalling in pulmonary fibrosis}

In humans, the FGF family has 22 structurally related family members. The most important ligands in the FGF family are FGF-1 and 2. FGFs interact with heparan sulfate glycosaminoglycans, and 18 out of the 22 known FGFs bind to the extracellular domains of FGFRs thereby inducing receptor activation [42]. The FGFR family has four members: FGFR-1, 2, 3 and 4. Each receptor consists of three extracellular immunoglobulin-type domains (D1-D3), a single-span transmembrane domain and an intracellular split tyrosine kinase domain [43]. FGFs and FGFRs regulate important cellular functions, such as cell proliferation, differentiation, migration and survival [44]. Once FGFRs recognise their FGF ligands, they undergo dimerisation followed by autophosphorylation and activation of downstream signalling via fibroblast growth factor receptor substrate 2, PI3K/protein kinase B (Akt), ERK1/2 and Ras/Raf/ mitogen-activated protein kinase (MAPK) pathways that are central to growth and cell survival (fig. 1) [45].

FGF-2 is a potent mitogen for fibroblasts [46], airway smooth muscle cells [47] and type II alveolar epithelial cells [48]. FGF-2 induces collagen synthesis in lung fibroblasts [46] and myofibroblasts [49]. Alveolar macrophages, fibroblasts, T-lymphocytes and endothelial cells are capable of producing FGF-2 [50-52]. However, in patients with IPF, mast cells seem to be the major source of FGF-2 [49]. Enhanced FGF-2 levels, as well as increased expression of FGFR-1 on epithelial, endothelial and smooth muscle cells/ myofibroblast-like cells, and increased expression of FGFR-2 on interstitial cells, were detected in the lungs of patients with IPF $[49,53]$. FGF-9 protein was increased in regions of active cellular hyperplasia, metaplasia and fibrotic expansion [54]. FGF-1 and FGF-10 might also have anti-fibrotic effects in the lung. Findings in human lung fibroblasts suggest that FGF-1 can reduce fibroblast growth rate, induce apoptosis of fibroblasts and inhibit differentiation into myofibroblasts [55]. FGF-10 has been shown to promote alveolar epithelial cell repair [56] and overexpression of FGF-10 attenuated bleomycin-induced pulmonary fibrosis in mice [57].

TGF- $\beta$ is a regulator of the FGF/FGFR signalling cascade, demonstrating the complex interactions among many growth factors. TGF- $\beta$ induces upregulation of FGFR-1 and FGF-2 in human lung fibroblasts [58] 
Fibrosis

(fibroblasts and myofibroblasts)
Angiogenesis

lendothelial cells, pericytes and vascular smooth muscle cells)

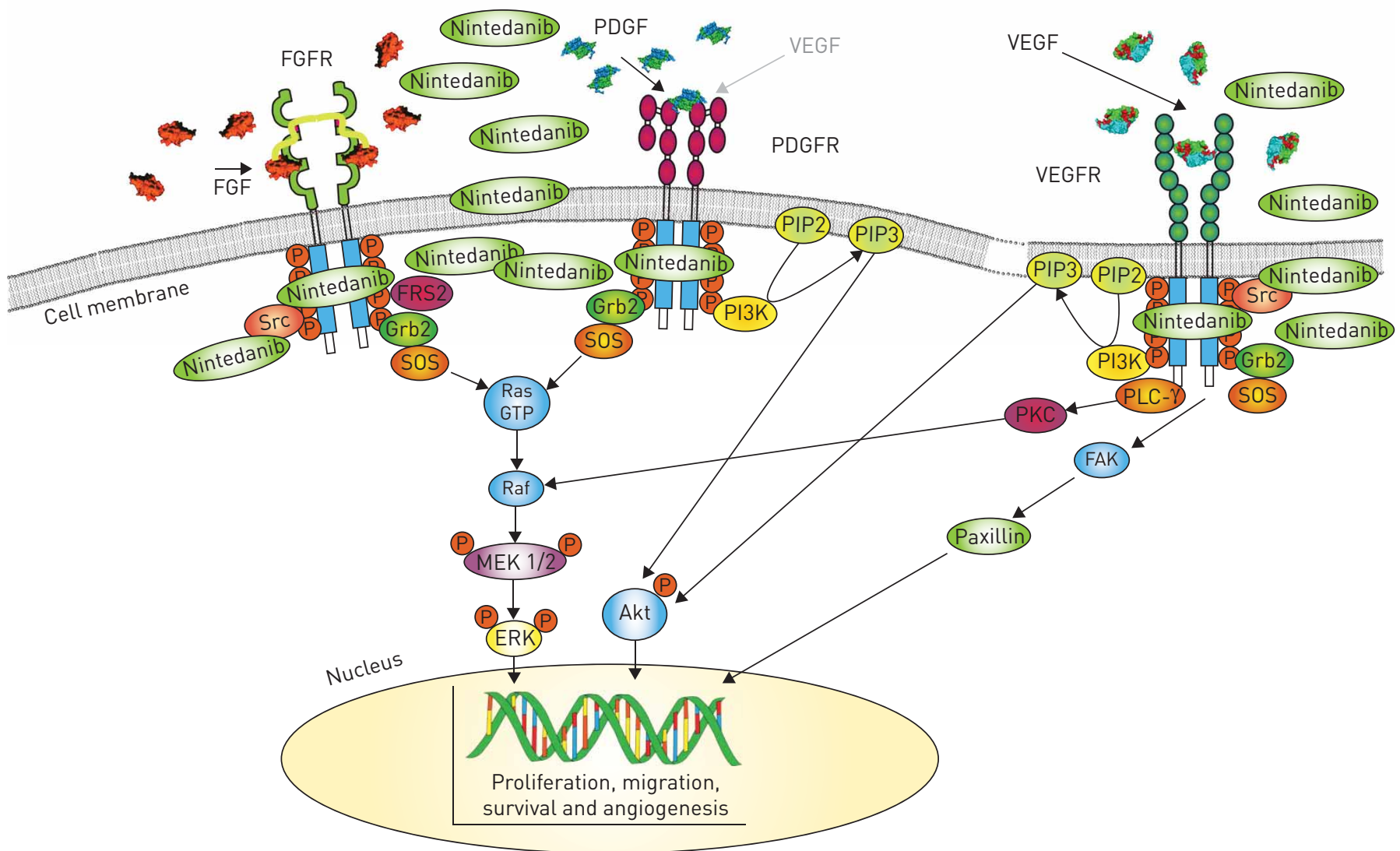

FIGURE 1 Polypharmacology of nintedanib and the downstream signalling pathways. Nintedanib binds to the intracellular ATP binding pocket of fibroblast growth factor receptors (FGFRs), platelet-derived growth factor receptors (PDGFRs) and vascular endothelial growth factor receptors (VEGFRs) resulting in blockage of the autophosphorylation of these receptors and the downstream signalling cascades. Nintedanib might exert additional activity by directly blocking non-receptor kinases like Src and Lck (not illustrated). Nintedanib was shown to inhibit PDGFR phosphorylation and subsequent protein kinase B (Akt) and extracellular signal-regulated kinase (ERK) $1 / 2$ phosphorylation in lung tissue from mice. Vascular endothelial growth factor (VEGF) was shown to stimulate angiogenesis via the VEGFR, but also to bind to the PDGFR on fibroblasts, stimulating proliferation. Inhibition by nintedanib ultimately results in reduced proliferation, migration and survival of fibroblasts and, potentially, also to attenuated angiogenesis in the lung. FAK: focal adhesion kinase; FGF: fibroblast growth factor; FRS2: FGFR substrate 2; Grb2: growth factor receptor-bound protein 2; MEK1/2: mitogen-activated protein kinase kinase 1/2; PDGF: platelet-derived growth factor; PI3K: phosphatidylinositol-4,5-bisphosphate 3-kinase; PIP2/3: phosphatidylinositol-2/3-phosphate; PKC: protein kinase C; PLC- $\gamma$ : phospholipase C- $\gamma$; SOS: son of sevenless, a guanine nucleotide exchange factor that acts on the Ras GTPases.

and an immediate release of FGF-2 from primary lung fibroblasts [59]. TGF- $\beta$-induced proliferation of pulmonary interstitial fibroblasts is blocked by an anti-FGF-2 antibody [59].

A role for FGF/FGFR signalling in animal models of lung fibrosis has also been shown. FGFR-1 is not detected in the lungs of healthy rats, but is strongly upregulated in a model of diffuse pulmonary fibrosis induced by paraquat plus hyperoxia [60]. In vivo, abrogation of FGF signalling reduced pulmonary fibrosis and improved survival in bleomycin-treated mice [61]. Taken together, the data suggest a prominent role of the FGF/FGFR signalling pathway in pulmonary fibrotic diseases.

\section{VEGF/VEGFR signalling in pulmonary fibrosis}

The vascular endothelial growth factor (VEGF) family consists of the structurally homologous secreted glycoproteins VEGF-A, B, C and D, and the placental growth factor [62]. VEGF-A and B play a key role in the regulation of blood vessel growth, while VEGF-C and D mainly regulate lymphangiogenesis [63]. The best characterised member of the VEGF family is VEGF-A [62]. The members of the VEGF family bind to the three VEGF receptors: VEGFR-1, 2 and 3. The VEGFRs are structurally similar, with an extracellular region, a transmembrane helix and a cytoplasmic region [62]. Activation of VEGFR leads to autophosphorylation of the receptor and downstream activation of signalling pathways including Ras, phospholipase C- $\gamma$, focal adhesion kinase, p38 and PI3K (fig. 1) [63]. The main sources of VEGF in the 
lungs are alveolar epithelial cells, bronchial epithelial cells, airway smooth muscle cells, fibroblasts, endothelial cells and alveolar macrophages $[64,65]$. VEGF-A has also been shown to stimulate PDGFRs, thereby regulating mesenchymal cell migration and proliferation (fig. 1) [66].

In patients with IPF, baseline plasma VEGF concentration was reported to be positively related to the fibrosis score on high-resolution computed tomography [67]. Furthermore, patients with IPF who developed progressive disease ( $\geqslant 10 \%$ decrease in FVC from baseline to month 6 ) had significantly higher plasma VEGF concentrations at baseline than non-progressors [67]. The 5-year survival rate tended to be worse in patients with high serum concentrations of VEGF compared with those with low concentrations [68]. In addition, serum VEGF levels appeared to predict deterioration of vital capacity in these patients. However, VEGF levels in the bronchoalveolar lavage fluid (BALF) are reported to be depressed in patients with IPF $[23,69,70]$. In a rat model of pulmonary fibrosis, VEGF expression was almost absent in fibrotic lesions, but strong in epithelial and endothelial cells [25]. Similarly, in patients with IPF, VEGF expression was low in fibroblasts and leukocytes in fibrotic lesions, but increased in capillary endothelial cells and alveolar type II epithelial cells in highly vascularised alveolar septa [22]. Experimental evidence in rats suggests that inhibition of VEGFR may reduce fibrosis [71] and that administration of VEGF aggravates the fibrogenic process [25]. However, VEGF was shown to have vasoprotective effects in the lung and to ameliorate pulmonary hypertension as a comorbidity of IPF $[24,25]$. Thus, the precise role of VEGF/ VEGFR signalling in IPF is controversial and remains to be further explored.

\section{Nintedanib is a potent inhibitor of the receptor tyrosine kinases PDGFR, FGFR and VEGFR}

Nintedanib is an indolinone derivative that was derived from a chemical lead optimisation programme designed for receptor tyrosine kinase inhibitors (Patent application WO2001027081, example 473). Nintedanib was originally designed as an anti-angiogenic drug targeting the receptor tyrosine kinases VEGFR, FGFR and PDGFR for the treatment of cancer [72]. Nintedanib is in phase II or III clinical development for several cancer indications (clinicaltrials.gov identifier numbers: NCT00805194, NCT00806819 and NCT01015118).

Nintedanib blocks kinase activity by occupying the intracellular ATP-binding pocket of specific tyrosine kinases. The binding mode was explored for FGFR-1 and VEGFR-2 (fig. 2, online supplementary material section A and fig. S1). Only subtle differences were found, which are discussed in online supplementary material section A.

The potency and selectivity of nintedanib were determined in enzymatic assays using human recombinant protein kinase domains [72]. Nintedanib is an inhibitor of the receptor tyrosine kinases PDGFR- $\alpha / \beta$ with

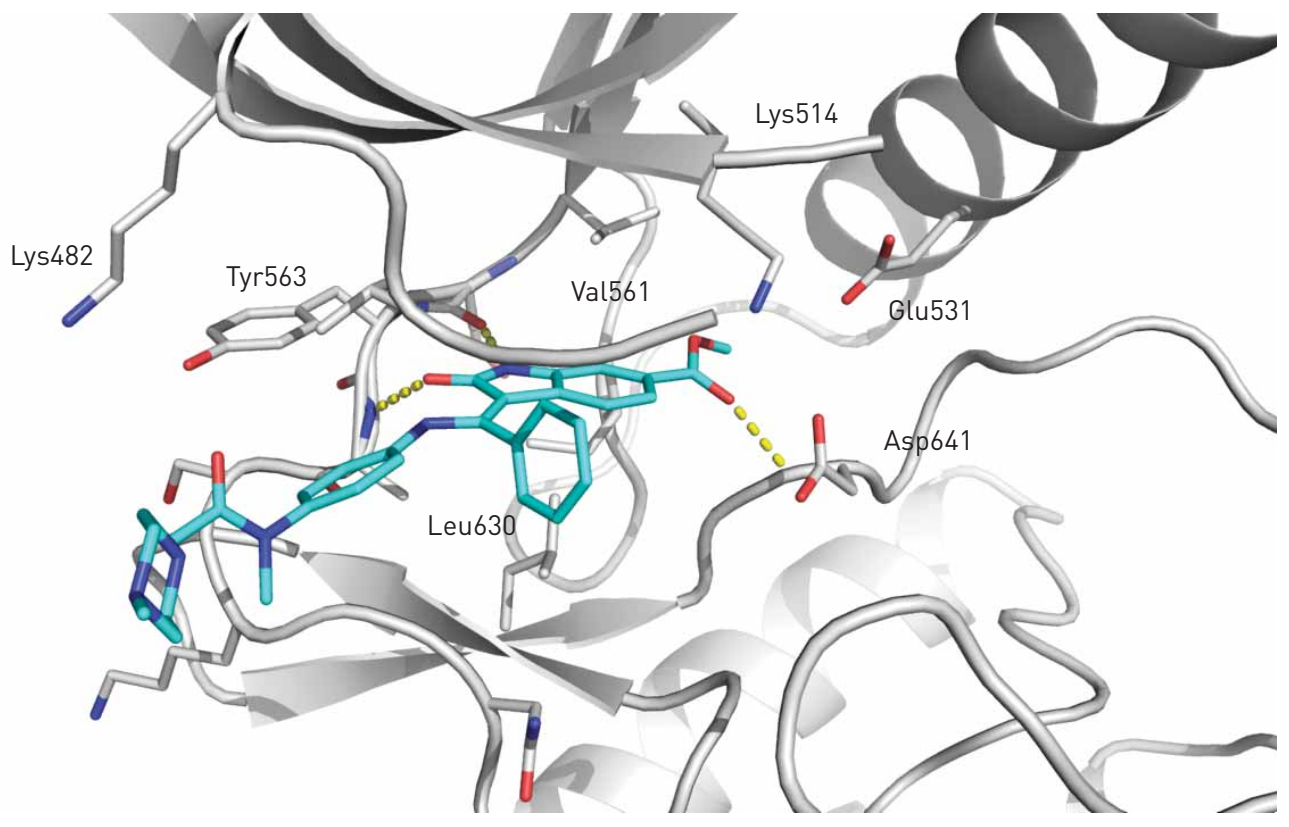

FIGURE 2 Crystal structure of nintedanib bound to the active site of the fibroblast growth factor receptor-1 kinase domain. The inhibitor and residues that line the active site are shown in stick representation. Hydrogen bonds are depicted as yellow dots. Key residues are labelled. 
half maximal inhibitory concentration $\left(\mathrm{IC}_{50}\right.$ ) values of 59 and $65 \mathrm{nmol} \cdot \mathrm{L}^{-1}$, respectively. Nintedanib inhibits FGFR-1, 2, 3 and 4 with $\mathrm{IC}_{50}$ values of $69,37,108$ and $610 \mathrm{nmol} \cdot \mathrm{L}^{-1}$, respectively, and VEGFR-1, 2 and 3 with $\mathrm{IC}_{50}$ values of 34,21 and $13 \mathrm{nmol} \cdot \mathrm{L}^{-1}$, respectively [72]. Nintedanib also inhibits FMS-like tyrosine kinase-3 (Flt-3) with an $\mathrm{IC}_{50}$ of $26 \mathrm{nmol} \cdot \mathrm{L}^{-1}$ [72]. Flt-3 is expressed on the surface of many haematopoietic progenitor cells and plays an important role in haematopoiesis [73].

Nintedanib also inhibits non-receptor tyrosine kinases of the Src family with $\mathrm{IC}_{50}$ values of $156 \mathrm{nmol} \cdot \mathrm{L}^{-1}$ for Src, $16 \mathrm{nmol} \cdot \mathrm{L}^{-1}$ for Lck and $195 \mathrm{nmol} \cdot \mathrm{L}^{-1}$ for Lyn [72]. Src is functionally involved in the control of a variety of cellular processes such as proliferation, differentiation, motility and adhesion. The Src family kinase inhibitor AZD0530 has been reported to exert anti-fibrotic effects in human lung fibroblasts and in bleomycin-induced lung fibrosis in mice [74]. Lck is functionally required for T-cell activation through the T-cell antigen receptor [75] and possibly required for T-cell survival [76]. Lyn is both positively and negatively involved in B-cell antigen receptor signalling, playing a role both in the initiation of the B-cell antigen receptor signal and in B-cell proliferation [77].

\section{Nintedanib interferes with essential fibrotic processes in a variety of in vitro assays and in vivo models \\ Effects on fibroblast proliferation and migration}

The inhibitory activity of nintedanib was confirmed at the cellular level in primary human lung fibroblasts from patients with IPF (IPF-HLF) and from control donors (N-HLF). Nintedanib inhibited PDGF-BB-stimulated PDGFR $\alpha$ and $\beta$ autophosphorylation in N-HLF with $\mathrm{IC}_{50}$ values of 22 and $39 \mathrm{nmol} \cdot \mathrm{L}^{-1}$, respectively, and PDGF-BB-stimulated proliferation in the same set of experiments with an $\mathrm{IC}_{50}$ of $64 \mathrm{nmol} \cdot \mathrm{L}^{-1}$ (fig. 3a) [78]. In IPF-HLF, PDGF-BB, FGF-2 and VEGF caused a significant pro-proliferative effect that was significantly reversed by nintedanib. In N-HLF, even lower concentrations of nintedanib significantly antagonised the growth factor-induced pro-proliferative effect. IPF-HLF demonstrated a stronger migratory response to all three growth factors compared to N-HLF. Growth factor-induced cell proliferation was significantly antagonised by nintedanib in both IPF-HLF and N-HLF [79]. In time lapse microscopy studies, nintedanib inhibited PDGF- and FGF-stimulated fibroblast motility in a concentration-dependent manner. Nintedanib attenuated PDGF-stimulated motility of IPF-HLF and N-HLF with $\mathrm{IC}_{50}$ values of 28 and $19 \mathrm{nmol} \cdot \mathrm{L}^{-1}$ and FGF-stimulated motility with $\mathrm{IC}_{50}$ values of 226 and $86 \mathrm{nmol} \cdot \mathrm{L}^{-1}$, respectively (fig. $3 \mathrm{~b}$ ).

\section{Effects on EMT}

Although epithelial cells are able to differentiate into mesenchymal cells [80], the importance of EMT in patients at the time of diagnosis and during further progression of IPF remains controversial [81]. Nintedanib at concentrations of $\geqslant 300 \mathrm{nmol} \cdot \mathrm{L}^{-1}$ did not change the morphology of primary alveolar type II epithelial cells derived from donors without IPF and showed no inhibitory activity on the TGF- $\beta$-induced ( $2 \mathrm{ng} \cdot \mathrm{mL}^{-1}$ ) EMT (online supplementary material section B).

a)

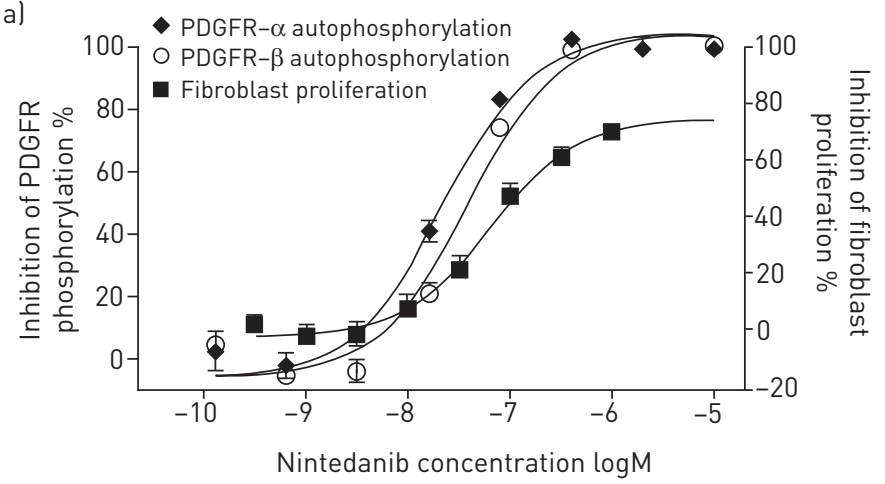

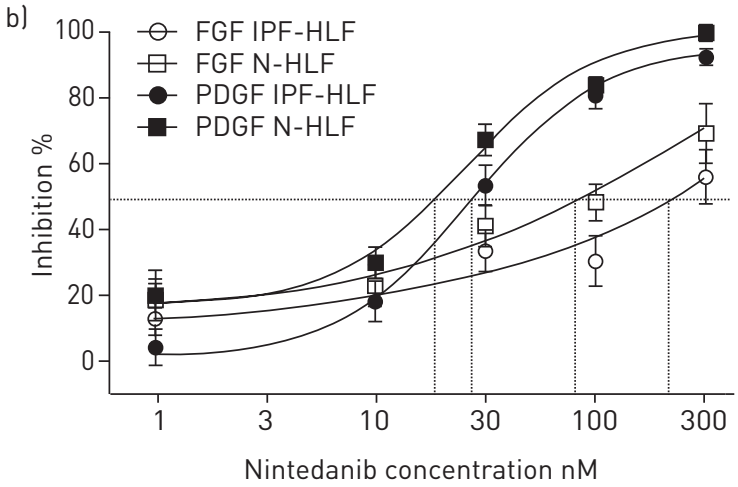

FIGURE 3 a) Nintedanib inhibits platelet-derived growth factor (PDGF)-BB-stimulated platelet-derived growth factor receptor (PDGFR)- $\alpha$ and $\beta$ autophosphorylation and proliferation of primary human lung fibroblasts (HLFs). HLFs were incubated with nintedanib at different concentrations and stimulated with PDGF-BB (50 ng.mL ${ }^{-1}$ ). PDGFR- $\alpha$ and $\beta$ phosphorylation was determined by an ELISA specific for the phosphorylated receptors. Proliferation was determined by bromodeoxyuridine incorporation. Concentration-dependent inhibition data are presented as mean \pm SEM ( $\mathrm{n}=3$ experiments). Reproduced from [78] with permission from the publisher. b) Nintedanib inhibits basic fibroblast growth factor (FGF) and PDGF-BB-stimulated motility of primary HLF from patients with idiopathic pulmonary fibrosis (IPF-HLF) and without IPF (N-HLF). Human lung fibroblasts were incubated with nintedanib at different concentrations for $30 \mathrm{~min}$ before the cells were stimulated with basic FGF $\left(20 \mathrm{ng} \cdot \mathrm{mL}^{-1}\right)$ or PDGF-BB $\left(50 \mathrm{ng} \cdot \mathrm{mL}^{-1}\right)$. Motility of the cells was determined by time lapse microscopy using a Cell-IQ imager (CM Technologies Oy, Tampere, Finland) and manual single cell tracking for $72 \mathrm{~h}$. Mean cell velocity was calculated from three experiments. Inhibition is presented as mean \pm SEM. 
Effects on fibroblast to myofibroblast transformation

Nintedanib inhibited TGF- $\beta$-induced $\left(10 \mathrm{ng} \cdot \mathrm{mL}^{-1}\right)$ fibroblast to myofibroblast transformation of primary human lung fibroblasts from IPF patients, as determined by $\alpha$-smooth muscle actin mRNA expression as a marker for myofibroblast differentiation, with an estimated $\mathrm{IC}_{50}$ value of $144 \mathrm{nmol} \cdot \mathrm{L}^{-1}[78]$.

\section{Effects on ECM components}

Nintedanib reduced TGF- $\beta$-stimulated collagen secretion and deposition by primary human lung fibroblasts from patients with IPF cultured for $48 \mathrm{~h}$. Nintedanib also reduced secreted TIMP-2 levels [78] and induced the secretion of pro-matrix metalloproteinase (MMP)-2 [79]. Reduced TIMP-2 levels, together with increased pro-MMP-2, could contribute to the reduction in collagen. Inhibition of fibroblast/myofibroblast proliferation by nintedanib might also exert an indirect inhibitory effect on ECM secretion and deposition.

\section{Effects on apoptosis}

In patients with IPF, lung fibroblasts/myofibroblasts are reported to have an elevated resistance to apoptotic stimulation preventing resolution of fibrosis [82]. Whether nintedanib is able to induce apoptosis in human lung fibroblasts is currently unknown. Nintedanib induced apoptosis in human umbilical vascular endothelial cells (HUVEC), human umbilical artery smooth muscle cells (HUASMC) and bovine retinal pericytes. Cleaved caspase- 3 was used as an indicator for apoptosis. In a concentration-dependent manner, nintedanib induced apoptosis in HUVECs stimulated with VEGF or FGF-2, in HUASMCs stimulated with PDGF-BB or FGF-2, and in pericytes stimulated with PDGF-BB and FGF-2 [72].

\section{Anti-angiogenic activity of nintedanib}

The potential impact of anti-angiogenic activity in the treatment of IPF has been discussed in the literature, but not yet clarified [26]. Experimental evidence has validated that inhibition of VEGF, PDGF and FGF signalling pathways reduces tumour angiogenesis in the lung [83]. Nintedanib inhibited proliferation of three cell types contributing to angiogenesis in the lung: endothelial cells, pericytes and smooth muscle cells [72]. Nintedanib inhibited the proliferation of VEGF-stimulated HUVECs, PDGF-BB-stimulated HUASMCs and PDGF-BB-stimulated bovine retinal pericytes with $\mathrm{IC}_{50}$ values of 9, 69 and $79 \mathrm{nmol} \cdot \mathrm{L}^{-1}$, respectively [72]. Furthermore, nintedanib reduced tumour microvessel density and demonstrated preclinical anti-angiogenic efficacy in an animal model of xenograft tumours in mice [72]. Taken together, the experimental evidence supports nintedanib having anti-angiogenic efficacy; however, whether this adds to its anti-fibrotic activity in IPF still needs to be elucidated.

\section{Anti-fibrotic and anti-inflammatory activity in animal models of lung fibrosis}

The in vivo efficacy of nintedanib was explored in three animal models of pulmonary fibrosis: bleomycin-induced lung fibrosis in mice and rats and silica-induced lung fibrosis in mice. The study settings and the treatment protocols are summarised in table 1 and the qualitative readouts are summarised in table 2 .

Briefly, PDGF-induced PDGFR phosphorylation as a receptor activation marker was determined by Western blotting of homogenates from mouse lung tissue. Animals were treated with nintedanib at different doses before receiving a single intratracheal dose of PDGF. The inhibitory activity of nintedanib on PDGFR phosphorylation was used to establish a pharmacokinetic/pharmacodynamic correlation for nintedanib in mice. The experiments confirmed the effective dose in mice to be in the range of $30-100 \mathrm{mg} \cdot \mathrm{kg}^{-1}$ [78].

\section{Effect on bleomycin-induced lung fibrosis in rats}

Nintedanib administered orally, once daily in a preventive treatment regimen starting at day 1 after intratracheal instillation of bleomycin inhibited lung fibrosis in the rat (online supplementary material

TABLE 1 Study settings and treatment protocols for the in vivo experiments in rats and mice

\begin{tabular}{|c|c|c|c|c|c|c|}
\hline Study & \multicolumn{4}{|c|}{ Bleomycin-induced lung fibrosis } & \multicolumn{2}{|c|}{$\frac{\text { Silica-induced lung fibrosis }}{\text { Mouse }}$} \\
\hline Posology & \multicolumn{2}{|c|}{ Once daily, oral } & \multicolumn{2}{|c|}{ Once daily, oral } & \multicolumn{2}{|c|}{ Once daily, oral } \\
\hline Dose $\mathbf{m g} \cdot \mathbf{k g}^{-1}$ & $10,30,50$ & 50 & 30,60 & 30,60 & 30,100 & 30,100 \\
\hline Study duration days & 21 & 21 & 14 & 21 & 30 & 30 \\
\hline Compound administration days & $0-21$ & $10-21$ & $0-14$ & $7-21$ & $0-30$ & $10-30,20-30$ \\
\hline
\end{tabular}


TABLE 2 Qualitative summary of the in vivo efficacy of nintedanib in animal models of lung fibrosis

\begin{tabular}{|c|c|c|c|c|c|c|}
\hline \multirow[b]{3}{*}{ Model } & \multicolumn{4}{|c|}{ Bleomycin-induced lung fibrosis } & \multirow{2}{*}{\multicolumn{2}{|c|}{$\begin{array}{c}\begin{array}{c}\text { Silica-induced lung } \\
\text { fibrosis }\end{array} \\
\text { Mouse }\end{array}$}} \\
\hline & \multicolumn{2}{|c|}{ Rat } & \multicolumn{2}{|c|}{ Mouse } & & \\
\hline & Preventive & Therapeutic & Preventive & Therapeutic & Preventive & Therapeutic ${ }^{\#}$ \\
\hline \multicolumn{7}{|c|}{ 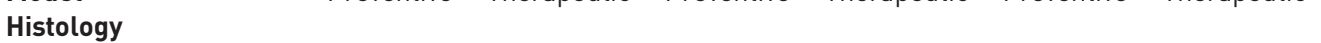 } \\
\hline Fibrosis & $\downarrow$ & $\downarrow$ & $\downarrow$ & $\downarrow$ & $\downarrow$ & $\downarrow$ \\
\hline Inflammation & ND & ND & $\downarrow$ & $\downarrow$ & $\downarrow$ & $\downarrow$ \\
\hline Granuloma & ND & ND & ND & ND & $\downarrow$ & $\downarrow$ \\
\hline \multicolumn{7}{|l|}{ Lung tissue } \\
\hline TGF- $\beta$ mRNA & $\downarrow$ & $\downarrow$ & ND & ND & ND & ND \\
\hline Procollagen-1 mRNA & $\downarrow$ & $\downarrow$ & ND & ND & ND & ND \\
\hline Total collagen & ND & ND & - & $\downarrow$ & $\downarrow$ & $\downarrow$ \\
\hline $\mathrm{IL}-1 \beta$ & ND & ND & $\downarrow$ & $\downarrow$ & $\downarrow$ & $\downarrow$ \\
\hline IL-6 & ND & ND & ND & ND & ND & $\downarrow$ \\
\hline CXCL1/KC & ND & ND & - & - & $\downarrow$ & $\downarrow$ \\
\hline TIMP-1 & ND & ND & $\downarrow$ & $\downarrow$ & $\downarrow$ & $\downarrow$ \\
\hline \multicolumn{7}{|l|}{ BALF } \\
\hline Total cells & ND & ND & - & - & - & - \\
\hline Macrophages & ND & ND & - & - & - & - \\
\hline Neutrophils & ND & ND & ND & ND & $\downarrow$ & $\downarrow$ \\
\hline Lymphocytes & ND & ND & $\downarrow$ & $\downarrow$ & $\downarrow$ & $\downarrow$ \\
\hline
\end{tabular}

$\downarrow$ : significant reduction (independent of dose used); -: no significant effect (independent of dose used); ND: not determined; TGF: transforming growth factor; IL: interleukin; TIMP: tissue inhibitors of metalloproteinase; BALF: bronchoalveolar lavage fluid. "\# : therapeutic treatment starting on day 10. Data from [78].

section C). In a dose-dependent manner, nintedanib resulted in only minimal inhibition, partial inhibition

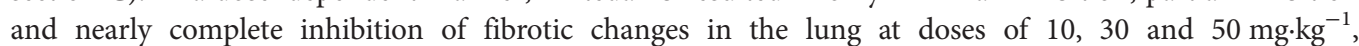
respectively. mRNA expression of fibrosis-related marker genes (TGF- $\beta 1$ and procollagen 1 ) was partially inhibited at $10 \mathrm{mg} \cdot \mathrm{kg}^{-1}$. At 30 and $50 \mathrm{mg} \cdot \mathrm{kg}^{-1}$ near complete inhibition was detected (table 2). When nintedanib was administered in a therapeutic regimen starting at day 10 , treatment with $50 \mathrm{mg} \cdot \mathrm{kg}^{-1}$ also resulted in a near complete attenuation of fibrosis as assessed histologically (fig. S2) and by gene expression of profibrotic markers (fig. S3).

\section{Effect on bleomycin-induced lung fibrosis in mice}

Preventative administration of nintedanib reduced bleomycin-induced lung inflammation regardless of the dose. This was demonstrated by reduced lymphocyte counts in the BALF, diminished IL-1 $\beta$ levels and a diminished percentage of myeloid dendritic cells in lung tissue. Furthermore, it reduced TIMP-1 and total collagen levels in lung tissue, and the fibrotic score in histomorphometric analysis of the lungs. In the therapeutic regimen, significant inhibitory effects of nintedanib on inflammation and fibrosis were also demonstrated [78].

Effect on silica-induced lung fibrosis in mice

Preventive administration of nintedanib once daily at 30 and $100 \mathrm{mg} \cdot \mathrm{kg}^{-1}$ by gavage reduced neutrophils and lymphocytes, but had no effect on macrophage counts in the BALF. Nintedanib significantly reduced IL-1 $\beta$, CXCL1/KC, TIMP-1 and total collagen in lung homogenates, and reduced lung inflammation, granuloma formation and fibrosis. Therapeutic administration starting at day 10 reduced neutrophils and lymphocytes in the BALF, total lung collagen and the fibrotic score in a comparable manner to preventive treatment. When treatment was started at day 20 , only lymphocytes in the BALF were significantly reduced. The reductions of IL-1 $\beta$, CXCL1/KC, TIMP-1, inflammatory score and granuloma score were smaller in the therapeutic regimen compared with the preventive regimen [78].

\section{Clinical efficacy}

The phase II, proof-of-concept TOMORROW study [84] suggested that 52 weeks of treatment with nintedanib $150 \mathrm{mg}$ twice daily in patients with IPF was associated with reductions in FVC decline and exacerbations, as well as preservation of health-related quality of life. Two phase III international, placebo-controlled, double-blind clinical studies with identical design (INPULSIS ${ }^{\oplus}-1$ and INPULSIS ${ }^{\bullet}-2$ ) 
investigated the efficacy and safety of nintedanib in patients with IPF [2, 3]. In total, 1066 patients from 24 countries were randomised three to two to receive nintedanib $150 \mathrm{mg}$ twice daily or placebo for 52 weeks, followed by a 4 -week follow-up period. The primary end-point was the annual rate of decline in FVC (in $\left.\mathrm{mL} \cdot \mathrm{year}^{-1}\right)$. Key secondary end-points were time to first acute exacerbation and change from baseline in the St George's Respiratory Questionnaire (SGRQ) total score over 52 weeks. Nintedanib consistently slowed disease progression by reducing the rate of decline in FVC compared with placebo. In INPULSIS -1 , the adjusted rate of decline in FVC was $114.7 \mathrm{~mL} \cdot \mathrm{year}^{-1}$ in the nintedanib group and $239.9 \mathrm{~mL} \cdot \mathrm{year}^{-1}$ in the placebo group, resulting in a difference between groups of $125.3 \mathrm{~mL} \cdot \mathrm{year}^{-1}(\mathrm{p}<0.0001)$. In INPULSIS ${ }^{\oplus}-2$, the adjusted rate of decline in FVC was $113.6 \mathrm{~mL} \cdot \mathrm{year}^{-1}$ in the nintedanib group and $207.3 \mathrm{~mL} \cdot \mathrm{year}^{-1}$ in the placebo group, resulting in a difference between groups of $93.7 \mathrm{~mL} \cdot \mathrm{year}^{-1}(\mathrm{p}=0.0002)$. The robustness and consistency of these results were confirmed by various sensitivity analyses, as well as additional FVC outcomes. In addition, the treatment effect on the primary outcome was consistent across a number of pre-specified subgroup analyses based on pooled data from the two INPULSIS ${ }^{\circledR}$ trials, which found a consistent treatment effect in subgroups defined by sex, age $(<65$ or $\geqslant 65$ years), race (White or Asian), baseline FVC $\%$ predicted $(\leqslant 70 \%$ or $>70 \%)$, baseline SGRQ total score $(\leqslant 40$ or $>40)$, smoking status (1) never-smoker or 2) current/ex-smoker), corticosteroids for systemic use at baseline (yes or no), and bronchodilator use at baseline (yes or no) [85]. The difference in mean change from baseline in SGRQ total score at week 52 was -2.69 points $(\mathrm{p}<0.02)$ in INPULSIS ${ }^{\oplus}-2$ and -0.05 points $(\mathrm{p}=0.97)$ in INPULSIS -1 .

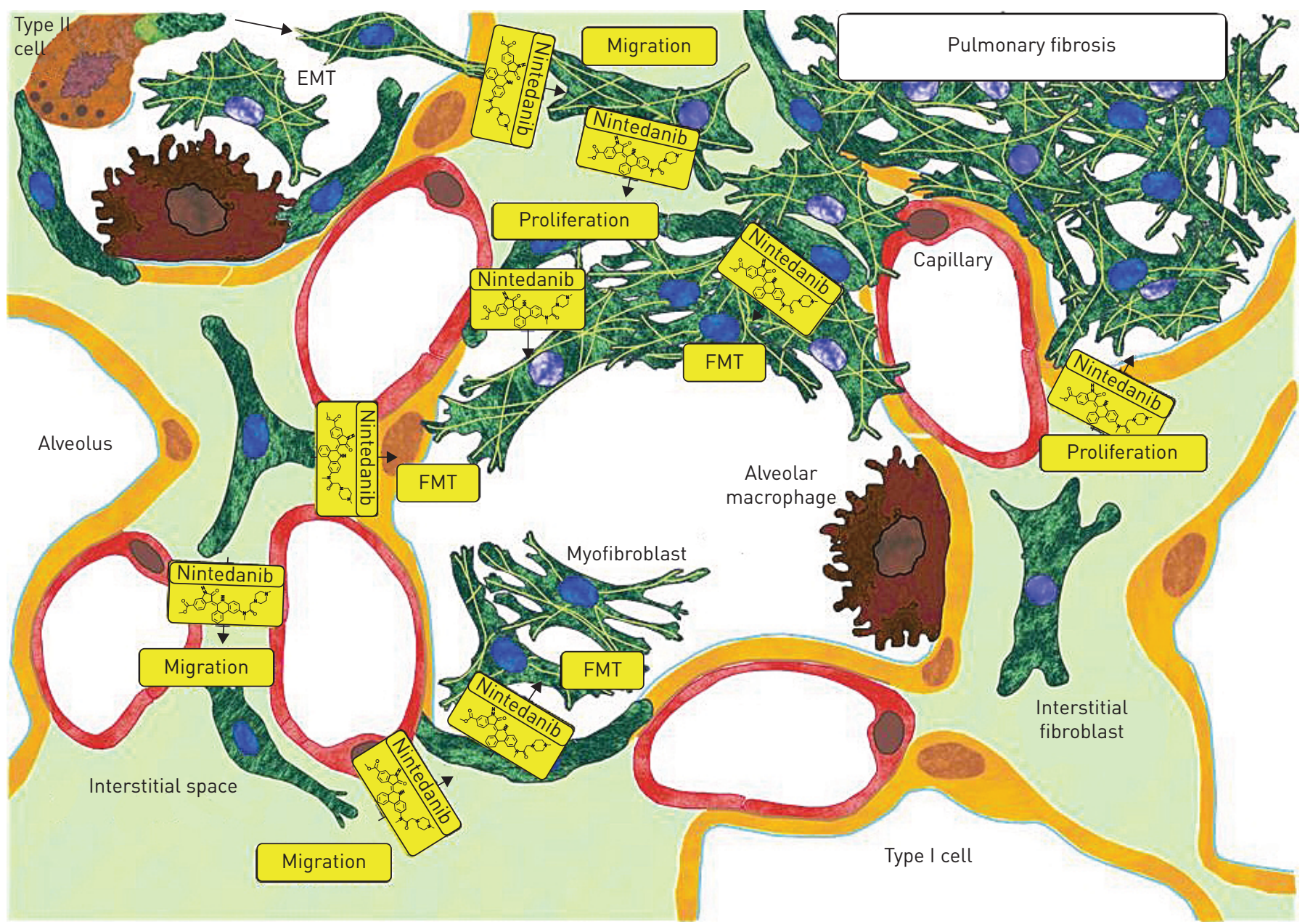

FIGURE 4 Current understanding of the mode of action of nintedanib in fibrotic lung diseases. The scheme reflects ongoing processes in the pathology of idiopathic pulmonary fibrosis (IPF). As a result of epithelial damage, alveolar epithelial cells undergo apoptosis and epithelial type II cells transform into myofibroblasts (epithelial-mesenchymal transition (EMT)) to provide mesenchymal cells for the initial repair process. Residual lung fibroblasts in the interstitium start to proliferate and migrate to the site of injury. Excessive fibroblast proliferation, migration and transformation to myofibroblasts (fibroblast to myofibroblast transformation (FMT)), and synthesis and deposition of extracellular matrix (ECM) are hallmarks of the fibrotic pathology in IPF. Nintedanib (yellow molecules) interferes with fibroblast/myofibroblast proliferation, FMT and migration. By limiting the number of fibroblasts/myofibroblasts, the synthesis and deposition of ECM are reduced. Nintedanib was found to have no effect on EMT. The effects of nintedanib on fibrocytes and other structural cells of the lung need further exploration. The inhibitory activities of nintedanib are shown in yellow. 
The proportion of patients with at least one acute exacerbation over 52 weeks was lower in the nintedanib group (3.9\%) compared with the placebo group $(9.6 \%)$ in INPULSIS $^{-}-2$, resulting in a hazard ratio (HR) of $0.38(\mathrm{p}=0.005)$, but no significant difference was observed in INPULSIS ${ }^{-1}$ (HR 1.15; $\mathrm{p}=0.67$ ). In a pre-specified sensitivity analysis of pooled data from the two trials on time to first confirmed or suspected exacerbation, as categorised by a blinded adjudication committee, there was a significantly reduced risk in patients treated with nintedanib versus placebo (HR 0.32; $\mathrm{p}=0.001$ ). The most commonly reported adverse event in both nintedanib groups was diarrhoea, affecting $~ 62 \%$ of patients (compared with $18 \%$ in the placebo groups). However, diarrhoea was mild or moderate in intensity in almost all cases and led to treatment discontinuation in fewer than $5 \%$ of patients treated with nintedanib.

\section{Summary and conclusion}

Nintedanib is an orally active small molecule tyrosine kinase inhibitor that has been evaluated in large clinical trials for the treatment of IPF, a fatal disease in which the uncontrolled proliferation of lung fibroblasts and deposition of ECM by myofibroblasts leads to progressive loss of lung function. This review describes the potent inhibitory activity of nintedanib in several in vitro assays investigating mechanisms present in the pathology of lung fibrosis. The polypharmacology of nintedanib on the receptors for FGF, PDGF and VEGF, and on non-receptor kinases like Src results in a broad inhibitory activity on the downstream signalling cascades of fibroblasts and myofibroblasts and, potentially also on cells involved in angiogenesis in the lung (fig. 1). Nintedanib exerted a potent inhibitory activity on the proliferation and migration of primary human lung fibroblasts (fig. 4). Additionally, at higher concentrations, nintedanib attenuated fibroblast to myofibroblast transformation and ECM deposition (fig. 4). Nintedanib acts primarily, but not exclusively, downstream of PDGF, FGF and VEGF, all of which are major growth factors in the pathogenesis of IPF. These cellular activities translated into consistent anti-fibrotic and anti-inflammatory activity in animal models of lung fibrosis, independent of the fibrosis-inducing stimulus. Taken together, these results provide a strong rationale for the clinical efficacy of nintedanib in IPF, which has recently been demonstrated in two replicate international phase III clinical trials.

\section{Acknowledgements}

Editorial assistance, supported financially by Boehringer Ingelheim, was provided by Wendy Morris of Fleishman-Hillard Group, Ltd, London, UK, during the preparation of this manuscript. The authors were fully responsible for all content and editorial decisions, and were involved at all stages of manuscript development and have approved the final version.

\section{References}

1 Vancheri C, Failla M, Crimi N, et al. Idiopathic pulmonary fibrosis: a disease with similarities and links to cancer biology. Eur Respir J 2010; 35: 496-504.

2 Richeldi L, Cottin V, Flaherty KR, et al. Design of the INPULSIS ${ }^{\mathrm{TM}}$ trials: two phase 3 trials of nintedanib in patients with idiopathic pulmonary fibrosis. Respir Med 2014; 108: 1023-1030.

3 Richeldi L, du Bois RM, Raghu G, et al. Efficacy and safety of nintedanib in idiopathic pulmonary fibrosis. $N$ Engl J Med 2014; 370: 2071-2082.

4 Raghu G, Collard HR, Egan JJ, et al. An official ATS/ERS/JRS/ALAT statement: idiopathic pulmonary fibrosis: evidence-based guidelines for diagnosis and management. Am J Respir Crit Care Med 2011; 183: 788-824.

5 American Thoracic Society, European Respiratory Society. American Thoracic Society/European Respiratory Society International Multidisciplinary Consensus Classification of the Idiopathic Interstitial Pneumonias. Am J Respir Crit Care Med 2002; 165: 277-304.

6 Nalysnyk L, Cid-Ruzafa J, Rotella P, et al. Incidence and prevalence of idiopathic pulmonary fibrosis: review of the literature. Eur Respir Rev 2012; 21: 355-361.

7 Olson AL, Swigris JJ. Idiopathic pulmonary fibrosis: diagnosis and epidemiology. Clin Chest Med 2012; 33: 41-50.

8 Noble PW, Albera C, Bradford WZ, et al. Pirfenidone in patients with idiopathic pulmonary fibrosis (CAPACITY): two randomised trials. Lancet 2011; 377: 1760-1769.

9 King TE Jr, Bradford WZ, Castro-Bernardini S, et al. A phase 3 trial of pirfenidone in patients with idiopathic pulmonary fibrosis. N Engl J Med 2014; 370: 2083-2092.

10 Borensztajn K, Crestani B, Kolb M. Idiopathic pulmonary fibrosis: from epithelial injury to biomarkers - insights from the bench side. Respiration 2013; 86: 441-452.

11 Selman M, King TE, Pardo A. Idiopathic pulmonary fibrosis: prevailing and evolving hypotheses about its pathogenesis and implications for therapy. Ann Intern Med 2001; 134: 136-151.

12 Steele MP, Schwartz DA. Molecular mechanisms in progressive idiopathic pulmonary fibrosis. Annu Rev Med 2013; 64: 265-276

13 Wuyts WA, Agostini C, Antoniou KM, et al. The pathogenesis of pulmonary fibrosis: a moving target. Eur Respir J 2013; 41: 1207-1218.

14 King TE Jr, Pardo A, Selman M. Idiopathic pulmonary fibrosis. Lancet 2011; 378: 1949-1961.

15 Bartis D, Mise N, Mahida RY, et al. Epithelial-mesenchymal transition in lung development and disease: does it exist and is it important? Thorax 2014; 69: 760-765.

16 Willis BC, duBois RM, Borok Z. Epithelial origin of myofibroblasts during fibrosis in the lung. Proc Am Thorac Soc 2006; 3: 377-382.

17 Maharaj S, Shimbori C, Kolb M. Fibrocytes in pulmonary fibrosis: a brief synopsis. Eur Respir Rev 2013; 22: $552-557$. 
Rowley JE, Johnson JR. Pericytes in chronic lung disease. Int Arch Allergy Immunol 2014; 164: 178-188.

Mubarak KK, Montes-Worboys A, Regev D, et al. Parenchymal trafficking of pleural mesothelial cells in idiopathic pulmonary fibrosis. Eur Respir J 2012; 39: 133-140.

2011; 16: 341-362.

Hecker L, Thannickal VJ. Nonresolving fibrotic disorders: idiopathic pulmonary fibrosis as a paradigm of impaired tissue regeneration. Am J Med Sci 2011; 341: 431-434.

Ebina M, Shimizukawa M, Shibata N, et al. Heterogeneous increase in CD34-positive alveolar capillaries in idiopathic pulmonary fibrosis. Am J Respir Crit Care Med 2004; 169: 1203-1208.

Cosgrove GP, Brown KK, Schiemann WP, et al. Pigment epithelium-derived factor in idiopathic pulmonary fibrosis: a role in aberrant angiogenesis. Am J Respir Crit Care Med 2004; 170: 242-251.

Farkas L, Gauldie J, Voelkel NF, et al. Pulmonary hypertension and idiopathic pulmonary fibrosis: a tale of angiogenesis, apoptosis, and growth factors. Am J Respir Cell Mol Biol 2011; 45: 1-15.

Farkas L, Farkas D, Ask K, et al. VEGF ameliorates pulmonary hypertension through inhibition of endothelial apoptosis in experimental lung fibrosis in rats. J Clin Invest 2009; 119: 1298-1311.

Renzoni EA. Neovascularization in idiopathic pulmonary fibrosis: too much or too little? Am J Respir Crit Care Med 2004; 169: 1179-1180.

Kelly M, Kolb M, Bonniaud P, et al. Re-evaluation of fibrogenic cytokines in lung fibrosis. Curr Pharm Des 2003; 9: 39-49.

Bonner JC. Regulation of PDGF and its receptors in fibrotic diseases. Cytokine Growth Factor Rev 2004; 15: 255-273.

29 Vaillant P, Menard O, Vignaud JM, et al. The role of cytokines in human lung fibrosis. Monaldi Arch Chest Dis 1996; 51: 145-152.

30 Adamali HI, Maher TM. Current and novel drug therapies for idiopathic pulmonary fibrosis. Drug Des Devel Ther 2012; 6: 261-272.

31 Clark JG, Madtes DK, Raghu G. Effects of platelet-derived growth factor isoforms on human lung fibroblast proliferation and procollagen gene expression. Exp Lung Res 1993; 19: 327-344.

32 Katzenstein AL, Myers JL. Idiopathic pulmonary fibrosis: clinical relevance of pathologic classification. Am J Respir Crit Care Med 1998; 157: 1301-1315.

33 Antoniades HN, Bravo MA, Avila RE, et al. Platelet-derived growth factor in idiopathic pulmonary fibrosis. J Clin Invest 1990; 86: 1055-1064.

34 Martinet Y, Rom WN, Grotendorst GR, et al. Exaggerated spontaneous release of platelet-derived growth factor by alveolar macrophages from patients with idiopathic pulmonary fibrosis. N Engl J Med 1987; 317: 202-209.

35 Abdollahi A, Li M, Ping G, et al. Inhibition of platelet-derived growth factor signaling attenuates pulmonary fibrosis. J Exp Med 2005; 201: 925-935.

36 Li M, Abdollahi A, Gröne HJ, et al. Late treatment with imatinib mesylate ameliorates radiation-induced lung fibrosis in a mouse model. Radiat Oncol 2009; 4: 66

37 Rice AB, Moomaw CR, Morgan DL, et al. Specific inhibitors of platelet-derived growth factor or epidermal growth factor receptor tyrosine kinase reduce pulmonary fibrosis in rats. Am J Pathol 1999; 155: 213-221.

38 Daniels CE, Wilkes MC, Edens M, et al. Imatinib mesylate inhibits the profibrogenic activity of TGF- $\beta$ and prevents bleomycin-mediated lung fibrosis. J Clin Invest 2004; 114: 1308-1316.

39 Aono Y, Nishioka Y, Inayama M, et al. Imatinib as a novel antifibrotic agent in bleomycin-induced pulmonary fibrosis in mice. Am J Respir Crit Care Med 2005; 171: 1279-1285.

40 Vuorinen K, Gao F, Oury TD, et al. Imatinib mesylate inhibits fibrogenesis in asbestos-induced interstitial pneumonia. Exp Lung Res 2007; 33: 357-373.

41 Daniels CE, Lasky JA, Limper $\mathrm{AH}$, et al. Imatinib treatment for idiopathic pulmonary fibrosis: randomized placebo-controlled trial results. Am J Respir Crit Care Med 2010; 181: 604-610.

42 Olsen SK, Garbi M, Zampieri N, et al. Fibroblast growth factor (FGF) homologous factors share structural but not functional homology with FGFs. J Biol Chem 2003; 278: 34226-34236.

43 Brooks AN, Kilgour E, Smith PD. Molecular pathways: fibroblast growth factor signaling: a new therapeutic opportunity in cancer. Clin Cancer Res 2012; 18: 1855-1862.

44 Boilly B, Vercoutter-Edouart AS, Hondermarck H, et al. FGF signals for cell proliferation and migration through different pathways. Cytokine Growth Factor Rev 2000; 11: 295-302.

45 Dey JH, Bianchi F, Voshol J, et al. Targeting fibroblast growth factor receptors blocks PI3K/AKT signaling, induces apoptosis, and impairs mammary tumor outgrowth and metastasis. Cancer Res 2010; 70: 4151-4162.

46 Chen Y, Zhao M, Fu M, et al. The role of calcineurin in the lung fibroblasts proliferation and collagen synthesis induced by basic fibroblast growth factor. Chin Med J (Engl) 2003; 116: 857-862.

47 Zou H, Nie XH, Zhang Y, et al. Effect of basic fibroblast growth factor on the proliferation, migration and phenotypic modulation of airway smooth muscle cells. Chin Med J (Engl) 2008; 121: 424-429.

48 Melloni B, Lesur O, Bouhadiba T, et al. Effect of exposure to silica on human alveolar macrophages in supporting growth activity in type II epithelial cells. Thorax 1996; 51: 781-786.

49 Inoue Y, King TE Jr, Tinkle SS, et al. Human mast cell basic fibroblast growth factor in pulmonary fibrotic disorders. Am J Pathol 1996; 149: 2037-2054.

50 Henke C, Marineili W, Jessurun J, et al. Macrophage production of basic fibroblast growth factor in the fibroproliferative disorder of alveolar fibrosis after lung injury. Am J Pathol 1993; 143: 1189-1199.

51 Rifkin DB, Moscatelli D. Recent developments in the cell biology of basic fibroblast growth factor. J Cell Biol 1989; 109: 1-6.

52 Blotnick S, Peoples GE, Freeman MR, et al. T lymphocytes synthesize and export heparin-binding epidermal growth factor-like growth factor and basic fibroblast growth factor, mitogens for vascular cells and fibroblasts: differential production and release by $\mathrm{CD}^{+}$and $\mathrm{CD}^{+}{ }^{+}$T cells. Proc Natl Acad Sci USA 1994; 91: 2890-2894.

53 Inoue Y, King TE Jr, Barker E, et al. Basic fibroblast growth factor and its receptors in idiopathic pulmonary fibrosis and lymphangioleiomyomatosis. Am J Respir Crit Care Med 2002; 166: 765-773.

54 Coffey E, Newman DR, Sannes PL. Expression of fibroblast growth factor 9 in normal human lung and idiopathic pulmonary fibrosis. J Histochem Cytochem 2013; 61: 671-679. 
55 Ramos C, Montaño M, Becerril C, et al. Acidic fibroblast growth factor decreases $\alpha$-smooth muscle actin expression and induces apoptosis in human normal lung fibroblasts. Am J Physiol Lung Cell Mol Physiol 2006; 291: L871-L879.

56 Upadhyay D, Bundesmann M, Panduri V, et al. Fibroblast growth factor-10 attenuates $\mathrm{H}_{2} \mathrm{O}_{2}$-induced alveolar epithelial cell DNA damage: role of MAPK activation and DNA repair. Am J Respir Cell Mol Biol 2004; 31: $107-113$.

57 Gupte VV, Ramasamy SK, Reddy R, et al. Overexpression of fibroblast growth factor-10 during both inflammatory and fibrotic phases attenuates bleomycin-induced pulmonary fibrosis in mice. Am J Respir Crit Care Med 2009; 180: 424-436.

58 Thannickal VJ, Aldweib KD, Rajan T, et al. Upregulated expression of fibroblast growth factor (FGF) receptors by transforming growth factor- $\beta 1$ (TGF- $\beta 1$ ) mediates enhanced mitogenic responses to FGFs in cultured human lung fibroblasts. Biochem Biophys Res Commun 1998; 251: 437-441.

59 Khalil N, Xu YD, O'Connor R, et al. Proliferation of pulmonary interstitial fibroblasts is mediated by transforming growth factor- $\beta 1$-induced release of extracellular fibroblast growth factor- 2 and phosphorylation of p38 MAPK and JNK. J Biol Chem 2005; 280: 43000-43009.

60 Barrios R, Pardo A, Ramos C, et al. Upregulation of acidic fibroblast growth factor during development of experimental lung fibrosis. Am J Physiol 1997; 273: L451-L458.

$61 \mathrm{Yu} \mathrm{ZH}$, Wang DD, Zhou ZY, et al. Mutant soluble ectodomain of fibroblast growth factor receptor-2 IIIc attenuates bleomycin-induced pulmonary fibrosis in mice. Biol Pharm Bull 2012; 35: 731-736.

62 Ferrara N, Gerber HP, LeCouter J. The biology of VEGF and its receptors. Nat Med 2003; 9: 669-676.

63 Koch S, Tugues S, Li X, et al. Signal transduction by vascular endothelial growth factor receptors. Biochem J 2011; 437: 169-183.

64 Mura M, dos Santos CC, Stewart D, et al. Vascular endothelial growth factor and related molecules in acute lung injury. J Appl Physiol (1985) 2004; 97: 1605-1617.

65 Tuder RM, Yun JH. Vascular endothelial growth factor of the lung: friend or foe. Curr Opin Pharmacol 2008; 8: 255-260.

66 Ball SG, Shuttleworth CA, Kielty CM. Vascular endothelial growth factor can signal through platelet-derived growth factor receptors. J Cell Biol 2007; 177: 489-500.

67 Simler NR, Brenchley PE, Horrocks AW, et al. Angiogenic cytokines in patients with idiopathic interstitial pneumonia. Thorax 2004; 59: 581-585.

68 Ando M, Miyazaki E, Ito T, et al. Significance of serum vascular endothelial growth factor level in patients with idiopathic pulmonary fibrosis. Lung 2010; 188: 247-252.

69 Koyama S, Sato E, Haniuda M, et al. Decreased level of vascular endothelial growth factor in bronchoalveolar lavage fluid of normal smokers and patients with pulmonary fibrosis. Am J Respir Crit Care Med 2002; 166: $382-385$.

70 Meyer KC, Cardoni A, Xiang ZZ. Vascular endothelial growth factor in bronchoalveolar lavage from normal subjects and patients with diffuse parenchymal lung disease. J Lab Clin Med 2000; 135: 332-338.

71 Hamada N, Kuwano K, Yamada M, et al. Anti-vascular endothelial growth factor gene therapy attenuates lung injury and fibrosis in mice. I Immunol 2005; 175: 1224-1231.

72 Hilberg F, Roth GJ, Krssak M, et al. BIBF 1120: triple angiokinase inhibitor with sustained receptor blockade and good antitumor efficacy. Cancer Res 2008; 68: 4774-4782.

73 Markovic A, MacKenzie KL, Lock RB. FLT-3: a new focus in the understanding of acute leukemia. Int J Biochem Cell Biol 2005; 37: 1168-1172.

74 Ding Q, Cai G-Q, Hu M, et al. Therapeutic targeting of Src kinase and myofibroblast activation in pulmonary fibrosis. Am J Respir Crit Care Med 2012; 185: A1944.

75 Nel AE. T-cell activation through the antigen receptor. Part 1: signaling components, signaling pathways, and signal integration at the T-cell antigen receptor synapse. J Allergy Clin Immunol 2002; 109: 758-770.

76 Seddon B, Legname G, Tomlinson P, et al. Long-term survival but impaired homeostatic proliferation of naïve T cells in the absence of p56lck. Science 2000; 290: 127-131.

77 DeFranco AL, Chan VW, Lowell CA. Positive and negative roles of the tyrosine kinase Lyn in B cell function. Semin Immunol 1998; 10: 299-307.

78 Wollin L, Maillet I, Quesniaux V, et al. Antifibrotic and anti-inflammatory activity of the tyrosine kinase inhibitor nintedanib in experimental models of lung fibrosis. J Pharmacol Exp Ther 2014; 349: 209-220.

79 Hostettler KE, Zhong J, Papakonstantinou E, et al. Anti-fibrotic effects of nintedanib in lung fibroblasts derived from patients with idiopathic pulmonary fibrosis. Respir Res 2014; 15: 157.

80 Marmai C, Sutherland RE, Kim KK, et al. Alveolar epithelial cells express mesenchymal proteins in patients with idiopathic pulmonary fibrosis. Am J Physiol Lung Cell Mol Physiol 2011; 301: L71-L78.

81 Rock JR, Barkauskas CE, Cronce MJ, et al. Multiple stromal populations contribute to pulmonary fibrosis without evidence for epithelial to mesenchymal transition. Proc Natl Acad Sci USA 2011; 108: E1475-E1483.

82 Thannickal VJ, Horowitz JC. Evolving concepts of apoptosis in idiopathic pulmonary fibrosis. Proc Am Thorac Soc 2006; 3: 350-356.

83 Crinò L, Metro G. Therapeutic options targeting angiogenesis in nonsmall cell lung cancer. Eur Respir Rev 2014; 23: 79-91.

84 Richeldi L, Costabel U, Selman M, et al. Efficacy of a tyrosine kinase inhibitor in idiopathic pulmonary fibrosis. N Engl J Med 2011; 365: 1079-1087.

85 Costabel U, Richeldi L, Azuma A, et al. Pre-specified subgroup analyses of pooled data from the INPULSISTM trials of nintedanib in idiopathic pulmonary fibrosis. The International Colloquium on Lung and Airway Fibrosis, 18th International Colloquium On Lung And Airway Fibrosis, 2014. www.iclaf.com/conference/index.php/2014/ ICLAF/paper/view/106. Date last accessed: November 3, 2014. Date last updated: August 6, 2014. 